\title{
Cyclodiode laser therapy for painful, blind glaucomatous eyes
}

\author{
Keith R G Martin, David C Broadway
}

\begin{abstract}
Aims-To determine the ability of cyclodiode laser treatment to relieve discomfort in painful blind glaucomatous eyes.

Methods-30 eyes underwent cyclodiode to reduce intraocular pressure (IOP) and relieve pain. Patients graded their precyclodiode and post-cyclodiode pain.

Results-After a minimum follow up of 6 months, a single cyclodiode treatment lowered mean IOP from $51 \mathrm{~mm} \mathrm{Hg}(95 \%$ CI plus or minus $3.7 \mathrm{~mm} \mathrm{Hg}$ ) to $26 \mathrm{~mm} \mathrm{Hg}$ (95\% CI plus or minus $5.8 \mathrm{~mm} \mathrm{Hg}$ ) providing pain relief in $73.3 \%(22 / 30)$. After retreatment of six eyes, mean IOP was reduced to $22(95 \%$ CI plus or minus 5.3) $\mathrm{mm} \mathrm{Hg}$ and pain relief was obtained in $96.7 \%(29 / 30)$. For eyes achieving pain relief after one treatment, IOP was reduced by $>30 \%$ in $81.0 \%(17 / 21)$. For eyes not achieving pain relief after one treatment, IOP was reduced by $>30 \%$ in only $22.2 \%(2 / 9)$ ( $p=0.0042$, Fisher's exact test). Conclusion-Cyclodiode was highly successful in providing pain relief in painful blind hypertensive glaucomatous eyes. The best predictor of successful pain relief was IOP reduction of $>\mathbf{3 0} \%$ from baseline. (Br F Ophthalmol 2001;85:474-476)
\end{abstract}

Cyclodiode laser treatment is being used increasingly to treat refractory glaucoma. ${ }^{1-8} \mathrm{In}$ patients with blind and painful eyes, perhaps the most important outcome measure is whether or not treatment renders the affected eye comfortable, yet few published studies have considered symptom relief as an outcome. The aim of the current study was to establish the ability of cyclodiode to improve comfort in patients with painful, blind eyes due to glaucoma. Our secondary aim was to establish which treatment related variables correlated with the achievement of a comfortable eye.

Department of Ophthalmology, Norfolk and Norwich Healthcare NHS Trust, West Norwich Hospital, Norwich, UK

K R G Martin D C Broadway

Correspondence to: David C Broadway, Department of Ophthalmology, West Norwich Hospital, Bowthorpe Road, Norwich, NR2 3TU, UK david.broadway@ norfolk.norwich.thenhs.com

Accepted for publication 24 October 2000
IOP following vitreoretinal surgery, and six had multiple pathologies. Informed consent was obtained in all cases. The aim of cyclodiode treatment was to achieve adequate IOP reduction and a comfortable eye. Minimum follow up was 6 months (mean 10.8 months, range 6-22 months). All patients were assessed 2-4 weeks after treatment. The frequency of further follow up was at the discretion of the clinician with a mean of 2.6 (1.13) visits between 1 month and 1 year.

Patients graded their ocular discomfort before and at each assessment after cyclodiode treatment. Pain was graded as "severe" (grade 3), "moderate" (grade 2), "mild" (grade 1), or "none" (grade 0). Topical and systemic treatment, visual acuity and IOP were recorded at each pretreatment and post-treatment visit.

Cyclodiode treatment was performed under peribulbar anaesthesia with $2 \%$ lignocaine using the OcuLight SLX semiconductor diode $810 \mathrm{~nm}$ laser (Iris Medical Instruments Inc, Mountain View, CA, USA) and the contact G-probe (Iris Medical Instruments). Treatment was delivered through a $600 \mu \mathrm{m}$ quartz fibre protruding $0.7 \mathrm{~mm}$ from the G-probe contact surface to indent the conjunctiva and sclera, thus improving energy delivery to the ciliary body. Transillumination was used to identify the ciliary body and 40 laser "shots" were applied, 10 in each quadrant of the ciliary body sparing the 3 and 9 o'clock positions. For first treatment sessions, the power of each shot was $1500 \mathrm{~mW}$ for $1500 \mathrm{~ms}$ (90 J per session). For retreatments, the duration of each shot was increased to $2000 \mathrm{~ms}$ (120 J per session). The criteria for retreatment were persistent pain and IOP $>30 \mathrm{~mm} \mathrm{Hg}$. Topical dexamethasone $0.1 \%$ was administered four times daily for 1 month after each cyclodiode treatment.

\section{Results}

CHANGE IN INTRAOCULAR PRESSURE (FIG 1)

The mean IOP recorded on the day of cyclodiode treatment was $51 \mathrm{~mm} \mathrm{Hg}(95 \%$ CI plus or minus $3.7 \mathrm{~mm} \mathrm{Hg}$, range $34-72 \mathrm{~mm} \mathrm{Hg}$ ). After a single cyclodiode treatment, the mean IOP fell to $26 \mathrm{~mm} \mathrm{Hg}$ (95\% CI plus or minus $5.8 \mathrm{~mm} \mathrm{Hg}$, range $1-66 \mathrm{~mm} \mathrm{Hg}$ ). At final follow up, after one $(n=23)$, two $(n=6)$, or three $(n=1)$ cyclodiode treatments, the mean IOP was $22 \mathrm{~mm} \mathrm{Hg}$ (95\% CI plus or minus $5.3 \mathrm{~mm}$ $\mathrm{Hg}$, range 1-66 $\mathrm{mm} \mathrm{Hg}$ ). Twenty two of 30 eyes $(73.3 \%)$ had an IOP reduction of greater than $30 \%$ with one treatment, and 27 of 30 eyes achieved a $30 \%$ reduction when retreatments were included.

PRE-CYCLODIODE AND POST-CYCLODIODE ANTIGLAUCOMATOUS THERAPY

Before cyclodiode treatment, five patients $(16.7 \%)$ were receiving oral acetazolamide treatment compared with two patients $(6.7 \%)$ 


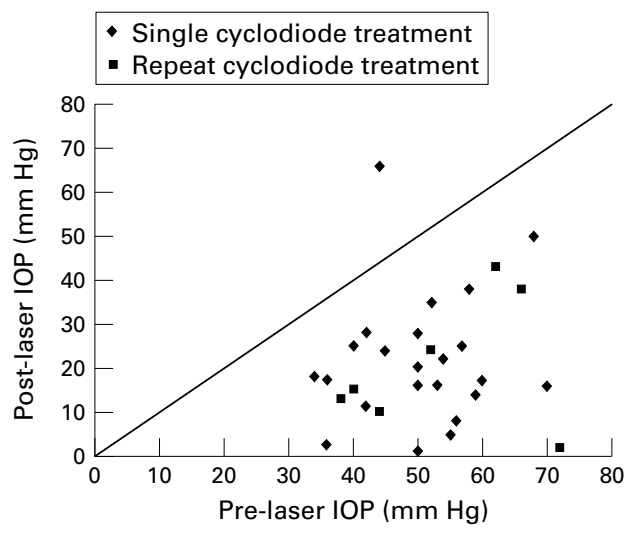

Figure 1 Pretreatment and post-treatment intraocular pressures following single and repeat cyclodiode therapy.

at final follow up. Twenty two eyes $(73.3 \%)$ were being treated with topical antiglaucomatous drugs before cyclodiode treatment compared with 12 eyes $(40.0 \%)$ at final follow up.

\section{PRE-CYCLODIODE AND POST-CYCLODIODE PAIN} SCORES

After a single cyclodiode, pain was reduced in 22 of 30 eyes $(73.3 \%)$ and abolished in 21 of 30 eyes $(70.0 \%)$. After retreatments, pain was reduced in 29 of 30 eyes $(96.7 \%)$ and abolished in 26 of 30 eyes (86.7\%) (Table 1). The only factor identified by multiple linear regression analysis (SPSS 6.1 for Windows, SPSS Inc, Chicago, IL, USA) to be associated with pain relief after a single treatment was IOP reduction $>30 \%$ from baseline $(p$ $<0.001$ ). Factors unassociated with successful pain relief included patient age, sex, ocular diagnosis, previous surgical/medical treatment, and pre-cyclodiode IOP and pain levels.

\section{COMPLICATIONS}

Complications occurred in six eyes. Three patients $(10.0 \%)$ developed persistent hypotony (IOP $<5 \mathrm{~mm} \mathrm{Hg}$ ), one hypotonous eye remained painful at final follow up but none developed phthisis. Post-treatment uveitis occurred in two eyes and transient hyphaema in one eye.

\section{Discussion}

We are aware of few studies on symptom control following cyclodiode treatment, although Walland et al reported pain control in six of eight painful, blind glaucomatous eyes using a treatment protocol similar to ours. ${ }^{7}$ We have demonstrated that cyclodiode treatment is very effective at relieving pain in patients with blind, painful, glaucomatous eyes. The treatment was well tolerated and only one of 30 eyes (3.3\%) remained painful at final follow up. Multiple

Table 1 Pre-cyclodiode and post-cyclodiode pain assessment

\begin{tabular}{|c|c|c|c|c|c|c|}
\hline & \multicolumn{2}{|c|}{ Pre-cyclodiode } & \multicolumn{2}{|c|}{ After single cyclodiode } & \multicolumn{2}{|c|}{$\begin{array}{l}\text { After repeat cyclodiode (if } \\
\text { necessary) }\end{array}$} \\
\hline & No & $\%$ & No & $\%$ & No & $\%$ \\
\hline No pain & 0 & 0.0 & 21 & 70.0 & 26 & 86.7 \\
\hline Mild pain & 0 & 0.0 & 1 & 3.3 & 2 & 6.7 \\
\hline Moderate pain & 24 & 80.0 & 7 & 23.3 & 2 & 6.7 \\
\hline Severe pain & 6 & 20.0 & 1 & 3.3 & 0 & 0.0 \\
\hline
\end{tabular}

linear regression analysis identified IOP reduction from baseline as the only variable correlating significantly with reduction in pain $(\mathrm{p}<0.001)$. The single most important factor in achieving complete pain relief with one treatment was a reduction in IOP of $>30 \%$ from baseline. For eyes achieving complete pain relief after one treatment, IOP was reduced by $>30 \%$ in $81.0 \%(17 / 21)$. For eyes not achieving pain relief after one treatment, IOP was reduced by $>30 \%$ in only $22.2 \%$ (2/9) $(\mathrm{p}=0.0042$, Fisher's exact test).

We used a very simple pain grading system, similar to the system used by Harmon, ${ }^{9}$ with patients classifying their pain as "severe" (grade 3), "moderate" (grade 2), "mild" (grade 1 ), or "none" (grade 0) at each assessment. This system was preferred to a "Visual analogue scale" system ${ }^{10} 11$ because we felt it was easier for patients to understand and was likely to be more repeatable.

We used a treatment protocol similar to that described by Bloom et $a l,{ }^{1}$ delivering $90 \mathrm{~J}$ to the ciliary body in the first treatment session and $120 \mathrm{~J}$ on retreatment. Spencer and Vernon $^{5}$ have recently reported good results using 14 applications of $2 \mathrm{~W}$ for 2 seconds (56 J per session) over 270 degrees; two of 58 eyes $(3.4 \%)$ developed hypotony (final IOP $<5 \mathrm{~mm}$ $\mathrm{Hg}$ ) compared with three of 30 eyes $(10.0 \%)$ in our study and three of $210(1.4 \%)$ in the study of Bloom. ${ }^{1}$ Our protocol involved a relatively high energy delivery to the ciliary body, which may explain the higher hypotony rate. However, only seven of our 30 (23.3\%) patients required retreatment compared with $45 \%,{ }^{5} 49 \%,{ }^{1}$ and $65 \%{ }^{12}$ in other published series. Our study included only patients with already "blind" eyes; the glaucoma in these eyes might therefore be expected to be more severe than other study populations. The pretreatment mean IOP of $51 \mathrm{~mm} \mathrm{Hg}$ in our series was also markedly higher than in the series of Bloom (34.1 mm Hg), ${ }^{1}$ Hawkins $(32.5 \%),{ }^{13}$ and Spencer $(33.0 \mathrm{~mm} \mathrm{Hg})^{5}$, consistent with more severe disease in patients with painful, blind eyes.

Other potential complications of cyclodiode treatment include malignant glaucoma, ${ }^{14}$ neurotrophic corneal defects, ${ }^{15}$ scleral perforation, ${ }^{16}$ and a theoretical risk of sympathetic ophthalmia. No such complications occurred in the present series.

\section{Conclusions}

Cyclodiode laser treatment very effectively eliminated discomfort in previously painful, blind, glaucomatous eyes. The best predictor of successful pain relief was reduction in IOP of $>30 \%$ from baseline. "Ideal" treatment parameters remain uncertain and protocols using slightly less total energy delivery to the ciliary body might have a lower risk of hypotony, although a corresponding increase in the need for retreatments might be expected.

Funding: Norwich Glaucoma Research Fund. Conflicting interests: none. 
1 Bloom PA, Tsai JC, Sharma K, et al. "Cyclodiode". Transscleral diode laser cyclophotocoagulation in the treatment of advanced refractory glaucoma. Ophthalmology 1997; 104:1508-19

2 Gupta N, Weinreb RN. Diode laser transscleral cyclophotocoagulation. F Glaucoma 1997;6:426-9.

3 Kosoko O, Gaasterland DE, Pollack IP, et al. Long-term outcome of initial ciliary ablation with contact diode laser transscleral cyclophotocoagulation for severe glaucoma. The Diode Laser Ciliary Ablation Study Group. Ophthalmology 1996;103:1294-302. $4 \begin{aligned} & \text { Montanari P, Italia A, Marangoni P, et al. Diode laser trans- } \\ & \text { scleral cyclophotocoagulation in refractory glaucoma treat- }\end{aligned}$ ment. Acta Ophthalmol Scand Suppl 1997;224:38.

5 Spencer AF, Vernon SA. "Cyclodiode": results of a standard protocol. Brf Ophthalmol 1999;83:311-6.

6 Threlkeld AB, Johnson MH. Contact transscleral diode cyclophotocoagulation for refractory glaucoma. f Glaucyclophotocoagulati

7 Walland MJ. Diode laser cyclophotocoagulation: dosestandardized therapy in end-stage glaucoma. Aust NZ F Standardized therapy in en

8 Yap-Veloso MI, Simmons RB, Echelman DA, et al. Intraocular pressure control after contact transscleral diode cyclophotocoagulation in eyes with intractable glaucoma. $\mathcal{7}$ Glaucoma 1998;7:319-28.

9 Harmon GK. Ultrasound therapy for treatment of glaucoma (cyclosclerotransthermy). In: El Sayyad F, Spaeth GL, Shields MB, Hitchings RA, eds. The refractory glaucomas. 1st ed. New York, Tokoyo: Igaku-Shoin, 1995:277-94.

10 Yarnitsky D, Sprecher E, Zaslansky R, et al. Multiple session experimental pain measurement. Pain 1996;67:327-33.

11 Todd KH, Funk KG, Funk JP, et al. Clinical significance of reported changes in pain severity. Ann Emerg Med 1996;27: 485-9.

12 Brancato R, Carassa RG, Bettin P, et al. Contact transscleral cyclophotocoagulation with diode laser in refractory glaucoma. Eur f Ophthalmol 1995;5:32-9.

13 Hawkins TA, Stewart WC. One-year results of semiconductor transscleral cyclophotocoagulation in patients with glaucoma. Arch Ophthalmol 1993;111:488-91.

14 Azuara-Blanco A, Dua HS. Malignant glaucoma after diode Azuara-Blanco A, Dua HS. Malignant glaucoma after diode
laser cyclophotocoagulation. Am $\mathcal{f}$ Ophthalmol 1999;127: laser cycior

15 Johnson SM. Neurotrophic corneal defects after diode laser cycloablation. Am f Ophthalmol 1998;126:725-7.

6 Sabri K, Vernon SA. Scleral perforation following transscleral cyclodiode. Br f Ophthalmol 1999;83:502-3. 